\title{
Glioma Stem Cells: Signaling, Microenvironment, and Therapy
}

\author{
Brandon D. Liebelt, ${ }^{1,2}$ Takashi Shingu, ${ }^{3}$ Xin Zhou, ${ }^{3}$ Jiangong Ren, ${ }^{3}$ \\ Seul A. Shin, ${ }^{3}$ and Jian $\mathrm{Hu}^{3}$ \\ ${ }^{1}$ Department of Neurosurgery, The University of Texas MD Anderson Cancer Center, Houston, TX 77030, USA \\ ${ }^{2}$ Department of Neurosurgery, Houston Methodist Neurological Institute, Houston, TX 77030, USA \\ ${ }^{3}$ Department of Cancer Biology, The University of Texas MD Anderson Cancer Center, Houston, TX 77030, USA \\ Correspondence should be addressed to Jian Hu; jhu3@mdanderson.org
}

Received 1 September 2015; Accepted 25 October 2015

Academic Editor: Ilya V. Ulasov

Copyright (c) 2016 Brandon D. Liebelt et al. This is an open access article distributed under the Creative Commons Attribution License, which permits unrestricted use, distribution, and reproduction in any medium, provided the original work is properly cited.

Glioblastoma remains the most common and devastating primary brain tumor despite maximal therapy with surgery, chemotherapy, and radiation. The glioma stem cell (GSC) subpopulation has been identified in glioblastoma and likely plays a key role in resistance of these tumors to conventional therapies as well as recurrent disease. GSCs are capable of self-renewal and differentiation; glioblastoma-derived GSCs are capable of de novo tumor formation when implanted in xenograft models. Further, GSCs possess unique surface markers, modulate characteristic signaling pathways to promote tumorigenesis, and play key roles in glioma vascular formation. These features, in addition to microenvironmental factors, present possible targets for specifically directing therapy against the GSC population within glioblastoma. In this review, the authors summarize the current knowledge of GSC biology and function and the role of GSCs in new vascular formation within glioblastoma and discuss potential therapeutic approaches to target GSCs.

\section{Introduction}

Glioblastoma is the most common and devastating primary brain tumor. The standard-of-care treatment involves maximal surgical resection followed by radiation and chemotherapy with temozolomide (TMZ) [1-4]. Despite treatment with rigorous surgical and medical therapy, patients only have a 15- to 19-month median overall survival rate because of near-universal tumor recurrence $[4,5]$. Studies have emerged showing glioma stem cells (GSCs) to represent a subpopulation of cells within glioblastoma that are characterized by increased resistance to chemotherapy and radiotherapy, suggesting that GSCs are likely responsible for failure of treatment and high recurrence rates in glioblastoma [6]. Therefore, GSCs are considered a relevant target for glioblastoma therapy, and the elimination of GSCs is crucial in treating glioblastoma. The strategy to target GSCs therapeutically is mainly focused on the direct ablation of GSCs by targeting cell surface markers and specific pathways that are required for maintaining GSC stemness. However, it has been increasingly acknowledged that another way to specifically target
GSCs is to alter the ability of GSCs to interact with their microenvironment, including their dependency on angiogenesis and their immune evasive properties. In this review, we summarize the current knowledge of GSC biology and function and discuss potential therapeutic approaches to target GSCs.

\section{Cancer Stem Cell Biology}

In their most basic definition, stem cells possess the ability to both self-renew and differentiate. Self-renewal is a critical function of stem cells, as they must persist throughout the entire lifespan of the organism. This quality of self-renewal is shared between both stem cells and cancer cells. Not surprisingly, there are several signaling pathways that have been identified, and likely numerous others yet to be identified, which are shared between these two cell types. Among these are the Notch, Sonic hedgehog (Shh), and Wnt signaling pathways [7]. These pathways are essential for preserving multipotency and self-renewal. 
Cancer stem cells (CSCs) possess the same characteristics of normal stem cells with the added features of being oncogenic in their host and giving rise to a heterogeneous population of cells that comprise the tumor mass. These cells were first postulated as etiologic agents in hematopoietic cancers. Bonnet and Dick showed that the leukemia-initiating cell in acute myeloid leukemia possessed the characteristics of a leukemia stem cell. This showed that normal primitive cells, rather than committed cells, were capable of leukemic transformation [8]. Since then, these cells have also been identified in solid tumors, including prostate [9], colon [10], lung [11], ovarian [12], and brain [13] tumors. A pure CSC tumor model posits that a CSC forms the basis for tumorigenesis and continued propagation through self-renewal and differentiation into the various cellular types that comprise the tumor [14].

Neural stem cells (NSCs) can be found in several locations in the adult brain including the subventricular zone (SVZ) [15], dentate gyrus of the hippocampus [16], and the subcortical white matter [17]. The SVZ is presumed to be host to the majority of these cells and has been proposed as the site of origin of gliomas and other brain tumors [18], stemming from early experiments showing increased tumor formation after carcinogen injection into the SVZ compared to other sites in rats [19].

Further, cell cultures derived from human glioblastoma have been shown to have the ability to form neurospheres. Cells constituting the neurospheres were found to highly express both Nestin and CD133. These cells were also capable of in vivo tumor formation when injected into nude mice, whereas non-sphere-forming cells isolated from glioblastoma did not grow tumor [20]. Additional studies investigating gliomagenesis by exposure to chemicals (ethylnitrosourea) or viruses (avian sarcoma virus) in animals showed that tumor formation preferentially occurs in the SVZ, particularly with earlier exposure to the carcinogen, suggesting the importance of this site in the origin of gliomas [9, 21-23].

\section{Implicated Signaling Pathways}

Compared to NSCs, GSCs exhibit enhanced self-renewal capacity and compromised differentiation [24], summarized in Figure 1. GSCs upregulate a number of signaling pathways required for maintaining NSC stemness, which enables them to enhance their stemness and aberrant cell survival, consequently leading to tumorigenesis [25-27]. Therefore, further understanding the signaling pathways in normal neural development including Notch, bone morphogenic proteins (BMPs), NF- $\kappa \mathrm{B}, \mathrm{Wnt}$, epidermal growth factor (EGF), and Shh will give significant insight into the cellular features of GSCs and will aid in designing better treatment strategies for glioblastoma.

Notch signaling is important for mediating various cellular and developmental processes including the regulation of proliferation, differentiation, apoptosis, and cell lineage decisions in NSCs [28-30]. Recent studies have implicated Notch signaling to be highly active in GSCs to suppress differentiation and maintain stem-like properties. Downregulation of Notch and its ligands such as Delta-like-1 and Jagged-1 leads to decrease in oncogenic potential of GSCs, which indicates an important role of Notch signaling in GSC survival and proliferation [28, 31, 32].

BMPs regulate proliferation, differentiation, and apoptosis in NSCs. BMP signaling pathways are activated in different developmental processes depending on their interaction with various signaling molecules including $\mathrm{Wnt} / \beta$-catenin, basic helix-loop-helix (bHLH), and hypoxia-inducible factor- $1 \alpha$ (HIF-1 $\alpha)$ [33-35]. Wnt signaling induces BMP expression, which predisposes NSCs toward an astroglial lineage [36]. Similarly, BMPs in GSCs are shown to play an important role in directing astroglial differentiation to inhibit the tumorigenic potential of GSCs [37]. Specifically, BMP-2 decreases GSC proliferation by directing astroglial differentiation and sensitizes GSCs to TMZ through destabilization of HIF- $1 \alpha$ $[34,38]$. In vivo delivery of BMP-4 inhibits brain tumor growth with a resultant decrease in mortality [37]. A BMP antagonist, Gremlin1, inhibits differentiation of GSCs by its regulation of endogenous BMP levels to maintain GSC selfrenewal and tumorigenic potential [39].

$\mathrm{Wnt} / \beta$-catenin signaling is also important for regulating NSC expansion and promoting astroglial lineage differentiation in normal neural development $[40,41]$. $\beta$-Catenin is a critical factor for proliferation and differentiation of GSCs $[42,43]$. Aberrant activation of Wnt signaling in GSCs leads to tumor growth through nuclear localization of stabilized $\beta$ catenin $[44,45]$. FoxM1/ $\beta$-catenin interaction regulates the transcription of $\mathrm{c}-\mathrm{Myc}$ and other Wnt target genes inducing glioma formation $[46,47]$. In addition, Wnt/ $\beta$-catenin signaling regulates the expression of PLAGL2 to suppress the differentiation of GSCs, maintaining their stemness [48].

The EGFR signaling pathway mediates proliferation, migration, differentiation, and survival in NSCs [49-53]. Levels of EGFR expression vary with specific stages of development, which suggests a requirement for precise modulation of EGFR expression by balancing extrinsic signals such as BMP and FGF during normal neuronal development [54]. EGFR activation promotes GSC proliferation and tumorigenesis by transactivation of $\beta$-catenin [55]. Furthermore, overexpression of EGFR increases the self-renewal capacity of GSCs resulting in induction of their tumorigenic potential [56-59].

Sonic hedgehog (Shh) signaling is pivotal in ventral patterning, proliferation, differentiation, and survival of NSCs [60-62]. In the adult brain, persistent Shh pathway signaling in the SVZ is critical for the regional specification and maintenance of NSCs $[63,64]$. Recent studies demonstrate that the Shh pathway is highly active in GSCs to maintain selfrenewal and induce tumorigenesis by regulating stemness genes. Suppression of Shh signaling reduces self-renewal and in vivo tumorigenicity, which indicates the dependency of GSCs on Shh signaling for their survival $[65,66]$.

\section{Microenvironment of GSCs: Vasculature in Glioma}

Neovascularization in malignant glioma is well documented, being characterized as hypervascular tumors associated with aberrant vascular morphology [67-69]. Normal vessels are formed by the mechanisms of vasculogenesis and angiogenesis [67, 70]. Vasculogenesis is in situ vascular formation 


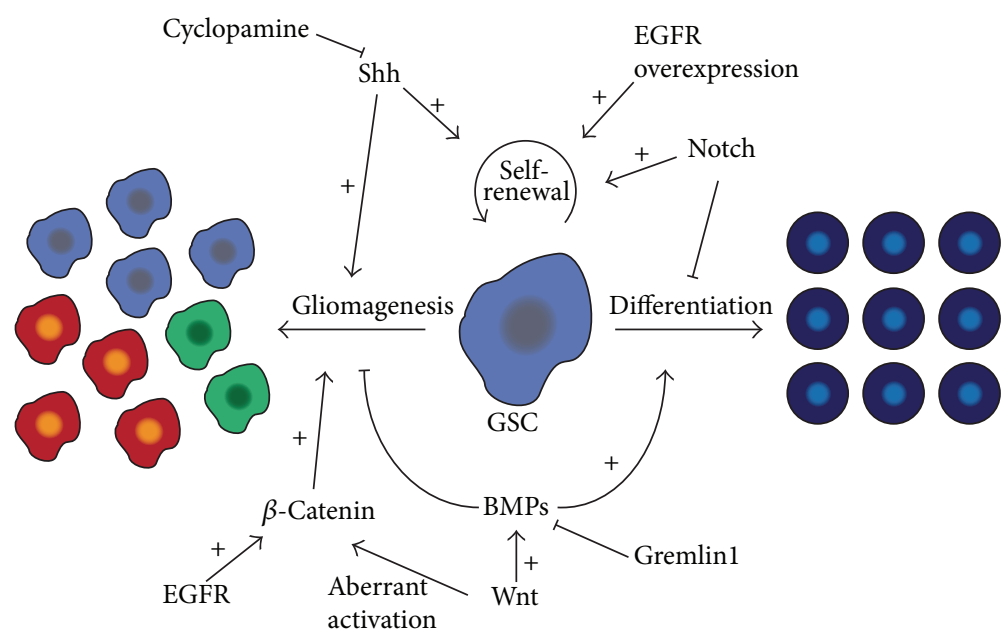

FIGURE 1: Summary of key molecular pathways regulating steps in glioma stem cell self-renewal, differentiation, and gliomagenesis.

through differentiation of mesodermally derived endothelial progenitor cells, angioblasts, which occurs during organogenesis and fetal development. Formation of a primitive vascular network by vasculogenesis is followed by angiogenesis, which contributes to expansion and remodeling of the existing vasculature by two different mechanisms: branching by vessel sprouting (sprouting angiogenesis) and splitting of vessel lumens by interstitial tissue (intussusception). Other than vasculogenesis and angiogenesis, malignant gliomas exhibit two additional types of neovascularization: vascular co-option and vasculogenic mimicry [67-69]. Recent studies implicate roles of GSCs in multiple modes of glioma neovascularization.

4.1. Vascular Co-Option. Glioma cells infiltrate around normal brain vessels forming perivascular cuffs, incorporating the existing vessels into the tumor in a process called vascular co-option [71]. Although the specific role of GSCs in vascular co-option has not been established, vascular co-option is followed by apoptosis of endothelial cells and regression of vessels. This results in hypoxia that in turn induces angiogenesis in which GSCs play critical roles [72].

4.2. Angiogenesis. Angiogenesis is the process of new vessel development from preexisting vasculature, with VEGF playing a critical role in this process [67]. Several studies suggest critical roles of GSCs in glioma angiogenesis. Conditioned medium from CD133+ GSCs contains 10-20-fold higher levels of VEGF than that from CD133- cells and promotes human microvascular endothelial cell migration and tube formation [73]. Hypoxia stimulates production of VEGF and stromal cell-derived factor 1 (SDF-1), also known as CXCL12, in GSCs [74, 75]. VEGF induces migration and proliferation of endothelial cells, while SDF-1 causes migration of endothelial cells [76]. GSCs also secrete hepatoma-derived growth factor that promotes endothelial cell migration in vitro and angiogenesis in vivo [77].
4.3. Vasculogenesis. Vasculogenesis was originally described as de novo vascular formation by angioblasts derived from mesoderm during organogenesis and fetal development, and it had been believed that postnatal vasculature could be formed only by angiogenesis even in pathological conditions $[69,70,78]$. This theory was challenged by findings of tumor vasculogenesis by Asahara et al., who reported formation of tumor vessels by circulating endothelial cell progenitors [79]. Several subsequent studies indicated that other types of cells including tumor-associated macrophages (TAMs)/Tie2 expressing monocytes and GSCs also differentiate into endothelial cells in the tumor [80-84]. Although there is controversy regarding what is referred to by "vasculogenesis," we will use vasculogenesis to describe any de novo neovascularization. VEGF and SDF-1 $\alpha$ were overexpressed by GSCrich C6 rat glioma cells in culture [76]. Inhibition of VEGF or SDF-1 $\alpha$ suppressed endothelial cell proliferation, tubule formation, and endothelial progenitor cell mobilization and decreased vascularization, suggesting an important role of GSCs in not only angiogenesis but also vasculogenesis. RicciVitiani et al. reported that some CD31+ endothelial cells in human glioblastoma specimens carried the same chromosomal aberrations as tumor cells. CD133+ GSCs cultured in endothelial conditions generated CD31+ and Tie$2+$ endothelial cells, and vessels in tumors formed by GSCs in immunocompromised mice were mainly composed of human CD31+ endothelial cells [83]. Wang et al. reported that glioblastoma-derived CD133+ cells included a CD144+ (vascular E-cadherin) population. These CD133/CD144 double positive cells showed an increase in expression of CD31, CD105, CD34, and VEGFR-2 and decrease in CD144 expression under endothelial culture conditions and were capable of forming a tubular network in Matrigel [85]. Finally, Soda et al. demonstrated that glioma tumor-initiating cells produced endothelial cells expressing CD31, CD34, CD144, and von Willebrand factor in a genetically engineered mouse brain tumor model [86]. Although the selection and interpretation of marker proteins for endothelial progenitor cells 
and endothelial cells are not identical, their findings suggest transdifferentiation of GSCs into endothelial cells. However, the biological and clinical significance of glioma vasculogenesis is still in debate. It was shown that only $10 \%$ of the vessels were identified to contain cells expressing neoplastic markers, and when identified these cells comprised less than $10 \%$ of the vascular cellularity in the cross section of human glioblastoma [87]. A study using chimeric mice with GFPtagged bone marrow cells also showed that less than $1 \%$ of bone marrow-derived cells were incorporated into the vascular endothelial layer in experimental gliomas [88]. Taken together, incorporation of bone marrow-derived or GSCderived cells into vascular endothelium may be a rare event or widely vary among tumors [67]. However, it is possible that vasculogenesis could play a critical role in glioma resistance to antiangiogenic therapy and early revascularization events in recurrent glioma [68].

Recent studies suggest that pericytes play a critical role not only in physiological processes such as wound healing but also in tumor growth and progression [89]. Furthermore, it was reported that GSCs give rise to pericytes [90, 91]. Although the role of GSC-derived pericytes in glioma neovascularization remains to be clarified, the finding that targeting GSC-derived pericytes suppressed neovasculature formation and tumor growth suggests an important function of these cells in glioma vascularization and progression.

4.4. Vasculogenic Mimicry. Vasculogenic mimicry is a fluidconducting, matrix-embedded meshwork that is formed not by endothelial cells but by tumor cells [92]. This finding has been observed in human malignant melanoma specimens [93] and documented in malignant astrocytoma [94, 95].

A study using human GSCs and GFP transgenic nude mice showed formation of patterned, tubular networks of vascular channels formed by human GSC-derived cells in xenograft tumors [96]. CD133+ GSCs established from human glioblastoma have successfully formed a vasculogenic network in a 3D Matrigel tube formation assay [97]. Similarly, Chen et al. demonstrated in vitro vascular formation by CD133+ GSCs with formation of vasculature lined by nonendothelial cells [98]. Knockdown of VEGFR-2 in GSCs inhibited formation of tubules, xenograft tumors, and vasculogenic mimicry [99]. In hypoxic conditions $\left(1 \% \mathrm{O}_{2}\right)$, CDH5 (CD144) was upregulated by HIF- $1 \alpha$ and HIF- $2 \alpha$ in GSCs and contributed to vasculogenic mimicry [100].

Although the extent of contribution of these mechanisms to glioma neovascularization seems to vary among tumors, GSCs are thought to contribute to at least three of the above four mechanisms. Additionally, GSCs can also transdifferentiate into pericytes that support the tumor vasculature.

\section{GSC-Dependent Therapeutic Resistance of Glioblastoma}

A major challenge for glioblastoma treatment is radioresistance and chemoresistance of the recurrent tumor, possibly due to an increased population of GSCs after initial treatment [101-103]. Increasing evidence shows that GSCs contribute to recurrence and therapy resistance through multiple mechanisms, such as alteration of DNA damage responsive machineries, hypoxic microenvironment, Notch signaling pathway, and multidrug-resistance mechanisms [6, 104-106]. On the other hand, these findings also offer a novel opportunity for therapeutic intervention on GSCs in glioblastoma patients.

Ionizing radiation represents an effective therapeutic option for glioblastoma by inducing DNA damage. Thus, DNA damage responses play crucial roles in cellular radiosensitivity and radioresistance [107]. To date, the underlying mechanism of radioresistance in glioblastoma remains elusive. Bao et al. observed that CD133+ GSCs were enriched after radiation, while CD133- cells were more sensitive to ionizing radiation [6]. Mechanistically, they found that CD133+ cells preferentially activated the DNA damage checkpoint in response to radiation and thus repaired DNA damage more efficiently [6]. Moreover, inhibitors of Chk1 and Chk2 checkpoint kinases could restore the radiosensitivity of CD133+ GSCs [6]. However, a study by McCord et al. showed conflicting results. They found that all six lines of CD133+ glioblastoma stem-like cells were more sensitive to radiation than the established glioma cell lines [108]. They also found that the CD133+ glioblastoma stem-like cells showed a significantly reduced DNA repair capacity [108]. One possible explanation for the contradictory observations between these two studies may be that established glioma cell lines (U87 and SF126), but not paired CD133- cells, were used as controls in McCord's study. TMZ, a commonly used alkylating agent, undergoes $\mathrm{pH}$-dependent hydrolysis to its reactive compound 5-3-(methyl)-1-(triazen-1-yl) imidazole-4carboxamide (MTIC) in cells, which causes DNA damage by methylating the O6-position or N7-position of guanine [109]. The methyl adducts lead to a continuous cycle of DNA base mismatch repair (MMR), resulting in double strand breaks and eventual apoptosis. Increasing evidence demonstrates that the O6-methylguanine methyltransferase (MGMT), whose function is repairing the mutagenic DNA lesion O6methylguanine back to guanine, is expressed in $80 \%$ of glioblastoma patients [110]. MGMT plays an important role in resistance to TMZ, and glioblastoma patients carrying a methylated MGMT promoter exhibit improved progressionfree and overall survival after treatment with alkylating agents $[110,111]$. Other signaling pathways such as (JNK) or microenvironment conditions (hypoxia) can also contribute to chemoresistance of glioblastoma by upregulating the expression of MGMT [112, 113]. Therefore, a better understanding of MGMT and DNA repair responses will help to delineate the detailed mechanisms of radioresistance and chemoresistance of GSCs.

A number of signaling pathways, including Notch, Shh, and receptor tyrosine kinase (RTK) signaling, have also been implicated in therapy resistance of glioblastoma. For example, $\gamma$-secretase inhibitors (GSIs) that inhibit the Notch pathway sensitize GSCs but not nonstem glioma cells to radiation [114]. In addition, overexpression of the constitutively active form of Notch1 or Notch2 rendered GSCs much more resistant to radiation [114]. A previous study has shown that ShhGLI signaling regulates the stemness of CD133+ GSCs, and cyclopamine, an inhibitor of Shh, displays synergistic effects 
with TMZ on GSC proliferation and apoptosis [66]. Another study confirmed that combination of either Notch inhibitor or hedgehog inhibitor, with temozolomide, enhanced the cytotoxicity on GSCs, and a significant effect was observed when the GSCs were treated with all three drugs simultaneously [115]. Abnormal activation of RTKs has been found in glioblastoma, such as PDGFR $\alpha$ in the proneural subtype and EGFR in the mesenchymal subtype [116]. Multiple RTKs and their involved signaling pathways are coactivated, leading to limited efficacy to therapy against single RTKs [117].

Environmental factors, like local cytokines and hypoxia, are crucial aspects of the microenvironment in glioblastoma and are generally correlated with worse prognosis. Among these extrinsic environmental factors, hypoxia has been attributed to play an important role in chemoresistance of GSCs. A recent study demonstrated that increased numbers of GSCs are localized in the core of the tumor mass along the intratumoral hypoxic gradient [118]. The hypoxic conditions help to maintain the undifferentiated state of GSCs through hypoxia-inducible factor- $2 \alpha$ (HIF- $2 \alpha$ ) and multiple HIF$2 \alpha$-induced genes $[119,120]$. Importantly, markers related to chemoresistance (TIMP-1 and MGMT) were also highly expressed in the GSCs of the inner tumor [118, 121]. Another study found that elevation of MGMT expression via HIF- $1 \alpha$ in GSCs contributes to its chemoresistance [113]. The observation that a hypoxia-driven undifferentiated state contributes to the chemoresistance of glioblastoma compels further effort to define the mechanisms of chemoresistance in GSCs and look for novel therapeutic approaches to target GSCs under the hypoxia niche effectively.

Recurrent glioblastoma exhibits resistance to multiple therapeutic drugs, leading to a hypothesis that GSCs are naturally resistant to chemotherapy. One potential explanation is that GSCs can reduce drug uptake or expel cytotoxic drugs by increasing the expression of ATP-binding cassette $(\mathrm{ABC})$ transporter [122]. A recent study suggested that the PTEN/PI3K/Akt pathway could regulate ABCG2 activity in glioma cancer stem-like cells [106]. The authors also showed that loss of PTEN or treatment with TMZ increased the GSC population [106]. Another possibility for chemoresistance of GSCs is that GSCs exhibit abnormalities of cell death pathways, such as overexpression of antiapoptotic proteins or downregulation of proapoptotic factors [123]. Further efforts need to be devoted to understanding the molecular mechanisms of chemoresistance in GSCs and developing novel and effective therapeutic approaches against GSCs.

\section{GSC-Targeted Therapies}

6.1. Therapeutic Targeting of GSCs by Surface Markers. CD133 is one of the best characterized cell surface makers in GSCs and NSCs. CD133+ cells in glioblastoma display cancer stem cell-like properties and CD133 is known to be highly expressed in GSCs [124]. Furthermore, patients with high levels of CD133 show poor clinical outcomes [125]. Thus, therapies against CD133 might represent a promising strategy for glioblastoma treatment. Brescia et al. reported that disruption of CD133 expression by short hairpin RNA in human glioblastoma neurospheres impaired the selfrenewal and tumorigenic capacity of neurosphere cells [124]. Further, treatment with carbon nanotubes conjugated to an anti-CD133 monoclonal antibody followed by irradiation with near-infrared laser light can selectively target CD133+ glioblastoma cells, and the photothermolysis caused by the nanotubes can kill targeted cells [126]. Recently, Emlet et al. reported that EGFRvIII is highly coexpressed with CD133 and EGFRvIII+/CD133+ defines the population of GSCs with the highest degree of self-renewal and tumor-initiating ability. Elimination of the EGFRvIII+/CD133+ population using a bispecific antibody could reduce tumorigenicity of implanted tumor cells, and the combined effect is better than any reagent directed against a single epitope [127].

L1 cell adhesion molecule (L1CAM, CD171) is a regulator of cell survival and is preferentially expressed on CD133+ GSCs $[128,129]$. Bao et al. reported that shRNA-mediated knockdown of L1CAM decreased the sphere-forming ability and induced apoptosis of CD133+, but not CD133-, glioma cells in vitro. L1CAM knockdown in CD133+ glioma cells prior to xenotransplantation into immunodeficient mice markedly inhibited in vivo tumorigenesis and prolonged survival of the xenograft recipients. Mechanistically, L1CAM knockdown decreased the expression of bHLH transcription factor and upregulated p21WAF1/CIP1 tumor suppresser in CD133+ glioma cells. Furthermore, intracranial administration of lentiviral shRNAs against L1CAM in glioma xenografts also substantially suppressed tumor growth and prolonged survival of the tumor-bearing mice [130]. Together, these data suggest that L1CAM is required for maintaining the growth and survival of CD133+ glioma cells both in vivo and in vitro, and L1CAM may represent a GSC specific therapeutic target for improving the treatment of glioblastoma and possibly other brain tumors.

However, despite these efforts relying on CD133 staining, recent studies indicate that $\mathrm{CD} 133$ + tumor cells cannot simply be considered GSCs because not all GSCs express CD133, and subgroups of glioblastoma driven by CD133- GSCs have recently been identified [131, 132]. Therefore, further work is needed to confirm the role of CD133 in GSCs and identify more optimal markers for GSCs. This will not only enhance our knowledge of GSCs but also give us additional understanding of effective ways to target these cells.

6.2. Therapeutic Targeting of GSCs by Signaling Pathway. Signaling pathways, such as Notch, Shh, VEGF, STAT3, and BMP, are important for regulating GSC self-renewal and differentiation. Therefore, targeting these signaling pathways and their receptors in GSCs holds promise for glioblastoma therapy. Notch signaling is known to promote the survival and proliferation of NSCs and to inhibit differentiation [133]. Fan et al. reported that inhibiting Notch activation by $\gamma$ secretase inhibitors (GSIs) resulted in diminished proliferation, increased neuronal differentiation, reduced CD133+ cell fraction in vitro, and decreased tumorigenicity in vivo [134]. Shh pathway is also highly expressed in both glioblastoma and cell lines, and Shh ligand is expressed in glioblastomaderived neurospheres. Treating glioblastoma-derived neurospheres with Shh inhibitor cyclopamine diminished new 
neurosphere formation, and viable glioblastoma cells injected intracranially following Shh blockade were no longer able to form tumors in athymic mice [65]. STAT3 pathway is required for GSC maintenance partially through upregulating TLR9 expression $[135,136]$. Herrmann et al. reported that stimulation of TLR9 with a CpG ligand (CpG ODN) activated STAT3 pathway signaling and promoted GSC growth, whereas silencing TLR9 expression abrogated GSC development [137].

Other than targeting the stemness signaling of GSCs, inducing GSCs differentiation is another approach that has been tested to target GSCs. Piccirillo et al. reported that activating BMP signaling could differentiate GSCs in experimental models of human glioblastoma [37]. Administration of BMP4 to human glioblastoma-bearing mice induced CD133+ GSC differentiation and markedly attenuated CD133+ GSC sphere-forming frequency [37]. In addition, implantation of BMP4-treated glioblastoma xenografts to murine recipients resulted in smaller tumor lesions and substantially prolonged host survival compared with untreated controls [37]. Therefore, BMP4 may act as a key inhibitory regulator of gliomagenesis and be used in combined stem cell-based therapy as a noncytotoxic therapeutic agent.

6.3. Therapeutic Targeting of the Tumor Microenvironment. Since the tumor microenvironment is essential for maintaining GSC stemness, targeting the microenvironment is a promising approach for treating glioblastoma. The glioblastoma microenvironment mainly is composed of microvasculature and TAMs. VEGF level has been recognized to correlate with microvasculature formation and tumor growth [138]. Recognition of the VEGF pathway as a key regulator of angiogenesis has led to the development of several VEGFtargeted agents such as bevacizumab. Calabrese et al. have treated mice bearing U87 glioma cell xenografts with bevacizumab and observed a reduction in the number of CD133+/ Nestin+ tumor-initiating cells, decreased microvasculature density, and decreased tumor growth [139]. Numerous studies have been reported showing that TAMs are enriched in glioblastoma and are very important components of the tumor microenvironment [140-144]. M2 TAMs could facilitate glioblastoma tumor growth by promoting neovascularization and play a tumor-supportive role in glioblastoma progression [145]. Recently, Zhou et al. reported that GSCs secrete periostin (POSTN) to recruit TAMs to support glioblastoma progression [146]. Silencing POSTN in GSCs markedly reduced TAM density, inhibited tumor growth, and increased survival of mice bearing GSC-derived xenografts. These studies indicate that targeting the interaction between GSCs and their microenvironment might represent an alternate approach in glioblastoma therapy.

\section{Conclusion}

In summary, glioblastoma remains a particularly challenging disease as little progress has been made towards improving patient outcomes and survival. A better understanding of the origins of this cancer and the molecular biology driving gliomagenesis is needed to tailor therapy towards addressing the root cause of this disease. Directly targeting glioma stem cells and their microenvironment presents a promising opportunity to eliminate the likely source of gliomas and the nidus of their recurrence.

\section{Conflict of Interests}

The authors declare that there is no conflict of interests regarding the publication of this paper.

\section{References}

[1] M. D. Walker, S. B. Green, D. P. Byar et al., "Randomized comparisons of radiotherapy and nitrosoureas for the treatment of malignant glioma after surgery," The New England Journal of Medicine, vol. 303, no. 23, pp. 1323-1329, 1980.

[2] L. M. DeAngelis, "Brain tumors," The New England Journal of Medicine, vol. 344, no. 2, pp. 114-123, 2001.

[3] R. Stupp, M. E. Hegi, W. P. Mason et al., "Effects of radiotherapy with concomitant and adjuvant temozolomide versus radiotherapy alone on survival in glioblastoma in a randomised phase III study: 5-year analysis of the EORTC-NCIC trial," The Lancet Oncology, vol. 10, no. 5, pp. 459-466, 2009.

[4] R. Stupp, W. P. Mason, M. J. Van Den Bent et al., "Radiotherapy plus concomitant and adjuvant temozolomide for glioblastoma," The New England Journal of Medicine, vol. 352, no. 10, pp. 987-996, 2005.

[5] S. A. Grossman and J. F. Batara, "Current management of glioblastoma multiforme," Seminars in Oncology, vol. 31, no. 5, pp. 635-644, 2004.

[6] S. Bao, Q. Wu, R. E. McLendon et al., "Glioma stem cells promote radioresistance by preferential activation of the DNA damage response," Nature, vol. 444, no. 7120, pp. 756-760, 2006.

[7] T. Reya, S. J. Morrison, M. F. Clarke, and I. L. Weissman, "Stem cells, cancer, and cancer stem cells," Nature, vol. 414, no. 6859, pp. 105-111, 2001.

[8] D. Bonnet and J. E. Dick, "Human acute myeloid leukemia is organized as a hierarchy that originates from a primitive hematopoietic cell," Nature Medicine, vol. 3, no. 7, pp. 730-737, 1997.

[9] A. T. Collins, P. A. Berry, C. Hyde, M. J. Stower, and N. J. Maitland, "Prospective identification of tumorigenic prostate cancer stem cells," Cancer Research, vol. 65, no. 23, pp. 10946-10951, 2005.

[10] L. Ricci-Vitiani, D. G. Lombardi, E. Pilozzi et al., "Identification and expansion of human colon-cancer-initiating cells," Nature, vol. 445, no. 7123, pp. 111-115, 2007.

[11] C. F. B. Kim, E. L. Jackson, A. E. Woolfenden et al., "Identification of bronchioalveolar stem cells in normal lung and lung cancer," Cell, vol. 121, no. 6, pp. 823-835, 2005.

[12] S. Zhang, C. Balch, M. W. Chan et al., "Identification and characterization of ovarian cancer-initiating cells from primary human tumors," Cancer Research, vol. 68, no. 11, pp. 4311-4320, 2008.

[13] S. K. Singh, I. D. Clarke, M. Terasaki et al., "Identification of a cancer stem cell in human brain tumors," Cancer Research, vol. 63, no. 18, pp. 5821-5828, 2003.

[14] S. J. Sundar, J. K. Hsieh, S. Manjila, J. D. Lathia, and A. Sloan, "The role of cancer stem cells in glioblastoma," Neurosurgical Focus, vol. 37, no. 6, article E6, 2014. 
[15] C. M. Morshead, B. A. Reynolds, C. G. Craig et al., "Neural stem cells in the adult mammalian forebrain: a relatively quiescent subpopulation of subependymal cells," Neuron, vol. 13, no. 5, pp. 1071-1082, 1994.

[16] P. S. Eriksson, E. Perfilieva, T. Björk-Eriksson et al., "Neurogenesis in the adult human hippocampus," Nature Medicine, vol. 4, no. 11, pp. 1313-1317, 1998.

[17] M. C. Nunes, N. S. Roy, H. M. Keyoung et al., "Identification and isolation of multipotential neural progenitor cells from the subcortical white matter of the adult human brain," Nature Medicine, vol. 9, no. 4, pp. 439-447, 2003.

[18] J. H. Globus and H. Kuhlenbeck, "The subependymal cell plate (matrix) and its relationship to brain tumors of the ependymal type," Journal of Neuropathology \& Experimental Neurology, vol. 3, no. 1, pp. 1-35, 1944.

[19] J. W. Hopewell and E. A. Wright, "The importance of implantation site in cerebral carcinogenesis in rats," Cancer Research, vol. 29, no. 11, pp. 1927-1931, 1969.

[20] X. Yuan, J. Curtin, Y. Xiong et al., "Isolation of cancer stem cells from adult glioblastoma multiforme," Oncogene, vol. 23, no. 58, pp. 9392-9400, 2004.

[21] D. D. Copeland and D. D. Bigner, "The role of the subependymal plate in avian sarcoma virus brain tumor induction: comparison of incipient tumors in neonatal and adult rats," Acta Neuropathologica, vol. 38, no. 1, pp. 1-6, 1977.

[22] N. A. Vick, M.-J. Lin, and D. D. Bigner, "The role of the subependymal plate in glial tumorigenesis," Acta Neuropathologica, vol. 40, no. 1, pp. 63-71, 1977.

[23] P. L. Lantos and D. J. Cox, "The origin of experimental brain tumours: a sequential study," Experientia, vol. 32, no. 11, pp. 1467-1468, 1976.

[24] J. Hu, A. L. Ho, L. Yuan et al., "Neutralization of terminal differentiation in gliomagenesis," Proceedings of the National Academy of Sciences of the United States of America, vol. 110, no. 36, pp. 14520-14527, 2013.

[25] H. D. Hemmati, I. Nakano, J. A. Lazareff et al., "Cancerous stem cells can arise from pediatric brain tumors," Proceedings of the National Academy of Sciences of the United States of America, vol. 100, no. 25, pp. 15178-15183, 2003.

[26] J. N. Rich and C. E. Eyler, "Cancer stem cells in brain tumor biology," Cold Spring Harbor Symposia on Quantitative Biology, vol. 73, pp. 411-420, 2008.

[27] A. L. Vescovi, R. Galli, and B. A. Reynolds, "Brain tumour stem cells," Nature Reviews Cancer, vol. 6, no. 6, pp. 425-436, 2006.

[28] S. Artavanis-Tsakonas, M. D. Rand, and R. J. Lake, "Notch signaling: cell fate control and signal integration in development," Science, vol. 284, no. 5415, pp. 770-776, 1999.

[29] P. Beatus and U. Lendahl, "Notch and neurogenesis," Journal of Neuroscience Research, vol. 54, no. 2, pp. 125-136, 1998.

[30] J. L. Lasky and H. Wu, "Notch signaling, brain development, and human disease," Pediatric Research, vol. 57, no. 5, pp. 104R-109R, 2005.

[31] M. Kanamori, T. Kawaguchi, J. M. Nigro et al., "Contribution of Notch signaling activation to human glioblastoma multiforme," Journal of Neurosurgery, vol. 106, no. 3, pp. 417-427, 2007.

[32] B. W. Purow, R. M. Haque, M. W. Noel et al., "Expression of Notch-1 and its ligands, Delta-like-1 and Jagged-1, is critical for glioma cell survival and proliferation," Cancer Research, vol. 65, no. 6, pp. 2353-2363, 2005.

[33] H. Kurooka, T. Nakahiro, K. Mori, K. Sano, and Y. Yokota, "BMP signaling is responsible for serum-induced Id2 expression,"
Biochemical and Biophysical Research Communications, vol. 420, no. 2, pp. 281-287, 2012.

[34] F. Pistollato, H.-L. Chen, B. R. Rood et al., "Hypoxia and HIF1 $\alpha$ repress the differentiative effects of BMPs in high-grade glioma," STEM CELLS, vol. 27, no. 1, pp. 7-17, 2009.

[35] Q. Weng, Y. Chen, H. Wang et al., "Dual-mode modulation of Smad signaling by Smad-interacting protein Sip1 is required for myelination in the central nervous system," Neuron, vol. 73, no. 4, pp. 713-728, 2012.

[36] M. Kasai, K. Satoh, and T. Akiyama, "Wnt signaling regulates the sequential onset of neurogenesis and gliogenesis via induction of BMPs," Genes to Cells, vol. 10, no. 8, pp. 777-783, 2005.

[37] S. G. M. Piccirillo, B. A. Reynolds, N. Zanetti et al., "Bone morphogenetic proteins inhibit the tumorigenic potential of human brain tumour-initiating cells," Nature, vol. 444, no. 7120, pp. 761-765, 2006.

[38] L. Persano, F. Pistollato, E. Rampazzo et al., "BMP2 sensitizes glioblastoma stem-like cells to Temozolomide by affecting HIF$1 \alpha$ stability and MGMT expression," Cell Death \& Disease, vol. 3, no. 10, article e412, 2012.

[39] K. Yan, Q. Wu, D. H. Yan et al., "Glioma cancer stem cells secrete Gremlinl to promote their maintenance within the tumor hierarchy," Genes \& Development, vol. 28, no. 10, pp. 1085-1100, 2014.

[40] S. Paina, D. Garzotto, S. DeMarchis et al., "Wnt5a is a transcriptional target of Dlx homeogenes and promotes differentiation of interneuron progenitors in vitro and in vivo," The Journal of Neuroscience, vol. 31, no. 7, pp. 2675-2687, 2011.

[41] Y. Pei, S. N. Brun, S. L. Markant et al., "WNT signaling increases proliferation and impairs differentiation of stem cells in the developing cerebellum," Development, vol. 139, no. 10, pp. 17241733, 2012.

[42] T. Grigoryan, P. Wend, A. Klaus, and W. Birchmeier, "Deciphering the function of canonical Wnt signals in development and disease: conditional loss- and gain-of-function mutations of $\beta$ catenin in mice," Genes \& Development, vol. 22, no. 17, pp. 23082341, 2008.

[43] R. N. Munji, Y. Choe, G. Li, J. A. Siegenthaler, and S. J. Pleasure, "Wnt signaling regulates neuronal differentiation of cortical intermediate progenitors," The Journal of Neuroscience, vol. 31, no. 5, pp. 1676-1687, 2011

[44] R. J. Atkins, J. Dimou, L. Paradiso et al., "Regulation of glycogen synthase kinase-3 beta (GSK-3 $\beta$ ) by the Akt pathway in gliomas," Journal of Clinical Neuroscience, vol. 19, no. 11, pp. 1558-1563, 2012.

[45] M. E. Hatten and M. F. Roussel, "Development and cancer of the cerebellum," Trends in Neurosciences, vol. 34, no. 3, pp. 134-142, 2011.

[46] A. Bowman and R. Nusse, "Location, location, location: FoxM1 mediates $\beta$-catenin nuclear translocation and promotes glioma tumorigenesis," Cancer Cell, vol. 20, no. 4, pp. 415-416, 2011.

[47] N. Zhang, P. Wei, A. Gong et al., "FoxM1 promotes $\beta$-catenin nuclear localization and controls Wnt target-gene expression and glioma tumorigenesis," Cancer Cell, vol. 20, no. 4, pp. 427442, 2011.

[48] H. Zheng, H. Ying, R. Wiedemeyer et al., "PLAGL2 regulates Wnt signaling to impede differentiation in neural stem cells and gliomas," Cancer Cell, vol. 17, no. 5, pp. 497-509, 2010.

[49] A. Ayuso-Sacido, J. A. Moliterno, S. Kratovac et al., "Activated EGFR signaling increases proliferation, survival, and migration and blocks neuronal differentiation in post-natal neural stem 
cells," Journal of Neuro-Oncology, vol. 97, no. 3, pp. 323-337, 2010.

[50] O. R. Lindberg, Å. Persson, A. Brederlau, A. Shabro, and H. G. Kuhn, "EGF-induced expansion of migratory cells in the rostral migratory stream," PLoS ONE, vol. 7, no. 9, Article ID e46380, 2012.

[51] R. Sanalkumar, S. Vidyanand, C. Lalitha Indulekha, and J. James, "Neuronal vs. glial fate of embryonic stem cell-derived neural progenitors (ES-NPs) is determined by FGF2/EGF during proliferation," Journal of Molecular Neuroscience, vol. 42, no. 1, pp. 17-27, 2010.

[52] J. Wang and R. K. Yu, "Interaction of ganglioside GD3 with an EGF receptor sustains the self-renewal ability of mouse neural stem cells in vitro," Proceedings of the National Academy of Sciences of the United States of America, vol.110, no. 47, pp. 1913719142, 2013.

[53] R. W. C. Wong, "Transgenic and knock-out mice for deciphering the roles of EGFR ligands," Cellular and Molecular Life Sciences, vol. 60, no. 1, pp. 113-118, 2003.

[54] L. Lillien and H. Raphael, "BMP and FGF regulate the development of EGF-responsive neural progenitor cells," Development, vol. 127, no. 22, pp. 4993-5005, 2000.

[55] W. Yang, Y. Xia, H. Ji et al., "Nuclear PKM2 regulates $\beta$-catenin transactivation upon EGFR activation," Nature, vol. 480, no. 7375, pp. 118-122, 2011.

[56] A. Ayuso-Sacido, C. Graham, J. P. Greenfield, and J. A. Boockvar, "The duality of epidermal growth factor receptor (EGFR) signaling and neural stem cell phenotype: cell enhancer or cell transformer?" Current Stem Cell Research \& Therapy, vol. 1, no. 3, pp. 387-394, 2006.

[57] F. Doetsch, L. Petreanu, I. Caille, J.-M. Garcia-Verdugo, and A. Alvarez-Buylla, "EGF converts transit-amplifying neurogenic precursors in the adult brain into multipotent stem cells," Neuron, vol. 36, no. 6, pp. 1021-1034, 2002.

[58] M. K. Nicholas, R. V. Lukas, N. F. Jafri, L. Faoro, and R. Salgia, "Epidermal growth factor receptor-mediated signal transduction in the development and therapy of gliomas," Clinical Cancer Research, vol. 12, no. 24, pp. 7261-7270, 2006.

[59] Y. Sun, S. K. Goderie, and S. Temple, "Asymmetric distribution of EGFR receptor during mitosis generates diverse CNS progenitor cells," Neuron, vol. 45, no. 6, pp. 873-886, 2005.

[60] S. Agarwala, T. A. Sanders, and C. W. Ragsdale, "Sonic hedgehog control of size and shape in midbrain pattern formation," Science, vol. 291, no. 5511, pp. 2147-2150, 2001.

[61] C. Chiang, Y. Litingtung, E. Lee et al., "Cyclopia and defective axial patterning in mice lacking Sonic hedgehog gene function," Nature, vol. 383, no. 6599, pp. 407-413, 1996.

[62] H. Roelink, J. A. Porter, C. Chiang et al., "Floor plate and motor neuron induction by different concentrations of the aminoterminal cleavage product of sonic hedgehog autoproteolysis," Cell, vol. 81, no. 3, pp. 445-455, 1995.

[63] F. Balordi and G. Fishell, "Hedgehog signaling in the subventricular zone is required for both the maintenance of stem cells and the migration of newborn neurons," The Journal of Neuroscience, vol. 27, no. 22, pp. 5936-5947, 2007.

[64] R. A. Ihrie, J. K. Shah, C. C. Harwell et al., "Persistent sonic hedgehog signaling in adult brain determines neural stem cell positional identity," Neuron, vol. 71, no. 2, pp. 250-262, 2011.

[65] E. E. Bar, A. Chaudhry, A. Lin et al., "Cyclopamine-mediated Hedgehog pathway inhibition depletes stem-like cancer cells in glioblastoma," Stem Cells, vol. 25, no. 10, pp. 2524-2533, 2007.
[66] V. Clement, P. Sanchez, N. de Tribolet, I. Radovanovic, and A. Ruiz i Altaba, "HEDGEHOG-GLI1 signaling regulates human glioma growth, cancer stem cell self-renewal, and tumorigenicity," Current Biology, vol. 17, no. 2, pp. 165-172, 2007.

[67] M. E. Hardee and D. Zagzag, "Mechanisms of glioma-associated neovascularization," The American Journal of Pathology, vol. 181, no. 4, pp. 1126-1141, 2012.

[68] N. Jhaveri, T. C. Chen, and F. M. Hofman, "Tumor vasculature and glioma stem cells: contributions to glioma progression," Cancer Letters, 2014.

[69] K. H. Plate, A. Scholz, and D. J. Dumont, "Tumor angiogenesis and anti-angiogenic therapy in malignant gliomas revisited," Acta Neuropathologica, vol. 124, no. 6, pp. 763-775, 2012.

[70] W. Risau, "Mechanisms of angiogenesis," Nature, vol. 386, no. 6626, pp. 671-674, 1997.

[71] J. Holash, P. C. Maisonpierre, D. Compton et al., "Vessel cooption, regression, and growth in tumors mediated by angiopoietins and VEGF,' Science, vol. 284, no. 5422, pp. 1994-1998, 1999.

[72] Y. Reiss, M. R. Machein, and K. H. Plate, “The role of angiopoietins during angiogenesis in gliomas," Brain Pathology, vol. 15, no. 4, pp. 311-317, 2005.

[73] S. Bao, Q. Wu, S. Sathornsumetee et al., "Stem cell-like glioma cells promote tumor angiogenesis through vascular endothelial growth factor," Cancer Research, vol. 66, no. 16, pp. 7843-7848, 2006.

[74] J. M. Heddleston, Z. Li, R. E. McLendon, A. B. Hjelmeland, and J. N. Rich, "The hypoxic microenvironment maintains glioblastoma stem cells and promotes reprogramming towards a cancer stem cell phenotype," Cell Cycle, vol. 8, no. 20, pp. 3274-3284, 2009.

[75] Y.-F. Ping, X.-H. Yao, J.-Y. Jiang et al., “The chemokine CXCL12 and its receptor CXCR4 promote glioma stem cell-mediated VEGF production and tumour angiogenesis via PI3K/AKT signalling," Journal of Pathology, vol. 224, no. 3, pp. 344-354, 2011.

[76] C. Folkins, Y. Shaked, S. Man et al., "Glioma tumor stemlike cells promote tumor angiogenesis and vasculogenesis via vascular endothelial growth factor and stromal-derived factor 1," Cancer Research, vol. 69, no. 18, pp. 7243-7251, 2009.

[77] C. Thirant, E.-M. Galan-Moya, L. G. Dubois et al., "Differential proteomic analysis of human glioblastoma and neural stem cells reveals HDGF as a novel angiogenic secreted factor," Stem Cells, vol. 30, no. 5, pp. 845-853, 2012.

[78] J. Folkman and Y. Shing, "Angiogenesis," The Journal of Biological Chemistry, vol. 267, no. 16, pp. 10931-10934, 1992.

[79] T. Asahara, T. Murohara, A. Sullivan et al., "Isolation of putative progenitor endothelial cells for angiogenesis," Science, vol. 275, no. 5302, pp. 964-966, 1997.

[80] C. E. Lewis, M. De Palma, and L. Naldini, “Tie2-expressing monocytes and tumor angiogenesis: regulation by hypoxia and angiopoietin-2," Cancer Research, vol. 67, no. 18, pp. 8429-8432, 2007.

[81] M. A. Venneri, M. De Palma, M. Ponzoni et al., "Identification of proangiogenic TIE2-expressing monocytes (TEMs) in human peripheral blood and cancer," Blood, vol. 109, no. 12, pp. 52765285, 2007.

[82] R. Du, K. V. Lu, C. Petritsch et al., "HIF1 $\alpha$ induces the recruitment of bone marrow-derived vascular modulatory cells to regulate tumor angiogenesis and invasion," Cancer Cell, vol. 13, no. 3, pp. 206-220, 2008. 
[83] L. Ricci-Vitiani, R. Pallini, M. Biffoni et al., “Tumour vascularization via endothelial differentiation of glioblastoma stem-like cells," Nature, vol. 468, no. 7325, pp. 824-828, 2010.

[84] L. Kilburn, M. F. Okcu, T. Wang et al., "Glutathione Stransferase polymorphisms are associated with survival in anaplastic glioma patients," Cancer, vol. 116, no. 9, pp. 22422249, 2010.

[85] R. Wang, K. Chadalavada, J. Wilshire et al., "Glioblastoma stemlike cells give rise to tumour endothelium," Nature, vol. 468, no. 7325, pp. 829-835, 2010.

[86] Y. Soda, T. Marumoto, D. Friedmann-Morvinski et al., "Transdifferentiation of glioblastoma cells into vascular endothelial cells," Proceedings of the National Academy of Sciences of the United States of America, vol. 108, no. 11, pp. 4274-4280, 2011.

[87] F. J. Rodriguez, B. A. Orr, K. L. Ligon, and C. G. Eberhart, "Neoplastic cells are a rare component in human glioblastoma microvasculature," Oncotarget, vol. 3, no. 1, pp. 98-106, 2012.

[88] M. R. Machein, S. Renninger, E. De Lima-Hahn, and K. H. Plate, "Minor contribution of bone marrow-derived endothelial progenitors to the vascularization of murine gliomas," Brain Pathology, vol. 13, no. 4, pp. 582-597, 2003.

[89] A. Armulik, G. Genové, and C. Betsholtz, "Pericytes: developmental, physiological, and pathological perspectives, problems, and promises," Developmental Cell, vol. 21, no. 2, pp. 193-215, 2011.

[90] L. Cheng, Z. Huang, W. Zhou et al., "Glioblastoma stem cells generate vascular pericytes to support vessel function and tumor growth," Cell, vol. 153, no. 1, pp. 139-152, 2013.

[91] P.-O. Guichet, S. Guelfi, M. Teigell et al., "Notch1 stimulation induces a vascularization switch with pericyte-like cell differentiation of glioblastoma stem cells," Stem Cells, vol. 33, no. 1, pp. 21-34, 2014.

[92] M. J. C. Hendrix, E. A. Seftor, A. R. Hess, and R. E. B. Seftor, "Vasculogenic mimicry and tumour-cell plasticity: lessons from melanoma," Nature Reviews Cancer, vol. 3, no. 6, pp. 411-421, 2003.

[93] A. J. Maniotis, R. Folberg, A. Hess et al., "Vascular channel formation by human melanoma cells in vivo and in vitro: vasculogenic mimicry," The American Journal of Pathology, vol. 155, no. 3, pp. 739-752, 1999.

[94] W.-Y. Yue and Z.-P. Chen, "Does vasculogenic mimicry exist in astrocytoma?" Journal of Histochemistry \& Cytochemistry, vol. 53, no. 8, pp. 997-1002, 2005.

[95] J.-M. Mao, J. Liu, G. Guo, X. Mao, and C. Li, "Glioblastoma vasculogenic mimicry: signaling pathways progression and potential anti-angiogenesis targets," Biomarker Research, vol. 3, no. 1, article 8, 2015.

[96] J. Dong, Q. Zhang, Q. Huang et al., "Glioma stem cells involved in tumor tissue remodeling in a xenograft model: laboratory investigation," Journal of Neurosurgery, vol. 113, no. 2, pp. 249260, 2010

[97] S. El Hallani, B. Boisselier, F. Peglion et al., "A new alternative mechanism in glioblastoma vascularization: tubular vasculogenic mimicry," Brain, vol. 133, no. 4, pp. 973-982, 2010.

[98] Y. Chen, Z. Jing, C. Luo et al., "Vasculogenic mimicry-potential target for glioblastoma therapy: an in vitro and in vivo study," Medical Oncology, vol. 29, no. 1, pp. 324-331, 2012.

[99] X. Yao, Y. Ping, Y. Liu et al., "Vascular endothelial growth factor receptor 2 (VEGFR-2) plays a key role in vasculogenic mimicry formation, neovascularization and tumor initiation by glioma stem-like cells," PLoS ONE, vol. 8, no. 3, Article ID e57188, 2013.
[100] X.-G. Mao, X.-Y. Xue, L. Wang et al., "CDH5 is specifically activated in glioblastoma stemlike cells and contributes to vasculogenic mimicry induced by hypoxia," Neuro-Oncology, vol. 15, no. 7, pp. 865-879, 2013.

[101] J. Chen, Y. Li, T.-S. Yu et al., "A restricted cell population propagates glioblastoma growth after chemotherapy," Nature, vol. 488, no. 7412, pp. 522-526, 2012.

[102] N. Sanai and M. S. Berger, "Glioma extent of resection and its impact on patient outcome," Neurosurgery, vol. 62, no. 4, pp. 753-764, 2008.

[103] B. Auffinger, D. Spencer, P. Pytel, A. U. Ahmed, and M. S. Lesniak, "The role of glioma stem cells in chemotherapy resistance and glioblastoma multiforme recurrence," Expert Review of Neurotherapeutics, vol. 15, no. 7, pp. 741-752, 2015.

[104] D. Hambardzumyan, M. Squartro, and E. C. Holland, "Radiation resistance and stem-like cells in brain tumors," Cancer Cell, vol. 10, no. 6, pp. 454-456, 2006.

[105] E. R. Blazek, J. L. Foutch, and G. Maki, "Daoy medulloblastoma cells that express CD133 are radioresistant relative to CD133cells, and the CD133+ sector is enlarged by hypoxia," International Journal of Radiation Oncology Biology Physics, vol. 67, no. 1, pp. 1-5, 2007.

[106] A.-M. Bleau, D. Hambardzumyan, T. Ozawa et al., "PTEN/ PI3K/Akt pathway regulates the side population phenotype and ABCG2 activity in glioma tumor stem-like cells," Cell Stem Cell, vol. 4, no. 3, pp. 226-235, 2009.

[107] B.-B. S. Zhou and S. J. Elledge, "The DNA damage response: putting checkpoints in perspective," Nature, vol. 408, no. 6811, pp. 433-439, 2000.

[108] A. M. McCord, M. Jamal, E. S. Williams, K. Camphausen, and P. J. Tofilon, "CD133+ glioblastoma stem-like cells are radiosensitive with a defective DNA damage response compared with established cell lines," Clinical Cancer Research, vol. 15, no. 16, pp. 5145-5153, 2009.

[109] K. Ochs and B. Kaina, "Apoptosis induced by DNA damage O6-methylguanine is Bcl-2 and caspase-9/3 regulated and Fas/caspase-8 independent," Cancer Research, vol. 60, no. 20, pp. 5815-5824, 2000.

[110] M. E. Hegi, A.-C. Diserens, T. Gorlia et al., "MGMT gene silencing and benefit from temozolomide in glioblastoma," The New England Journal of Medicine, vol. 352, no. 10, pp. 997-1003, 2005.

[111] M. E. Hegi, L. Liu, J. G. Herman et al., "Correlation of $\mathrm{O}^{6}$ methylguanine methyltransferase (MGMT) promoter methylation with clinical outcomes in glioblastoma and clinical strategies to modulate MGMT activity," Journal of Clinical Oncology, vol. 26, no. 25, pp. 4189-4199, 2008.

[112] M. Okada, A. Sato, K. Shibuya et al., "JNK contributes to temozolomide resistance of stem-like glioblastoma cells via regulation of MGMT expression," International Journal of Oncology, vol. 44, no. 2, pp. 591-599, 2014.

[113] L. Persano, F. Pistollato, E. Rampazzo et al., "BMP2 sensitizes glioblastoma stem-like cells to Temozolomide by affecting HIFlalpha stability and MGMT expression," Cell Death and Disease, vol. 3, article e412, 2012.

[114] J. Wang, T. P. Wakeman, J. D. Lathia et al., "Notch promotes radioresistance of glioma stem cells," Stem Cells, vol. 28, no. 1, pp. 17-28, 2010.

[115] I. V. Ulasov, S. Nandi, M. Dey, A. M. Sonabend, and M. S. Lesniak, "Inhibition of sonic hedgehog and notch pathways enhances sensitivity of $\mathrm{CD} 33^{+}$glioma stem cells to temozolomide therapy," Molecular Medicine, vol. 17, no. 1-2, pp. 103-112, 2011. 
[116] R. G. W. Verhaak, K. A. Hoadley, E. Purdom et al., "Integrated genomic analysis identifies clinically relevant subtypes of glioblastoma characterized by abnormalities in PDGFRA, IDH1, EGFR, and NF1," Cancer Cell, vol. 17, no. 1, pp. 98-110, 2010.

[117] J. M. Stommel, A. C. Kimmelman, H. Ying et al., "Coactivation of receptor tyrosine kinases affects the response of tumor cells to targeted therapies," Science, vol. 318, no. 5848, pp. 287-290, 2007.

[118] F. Pistollato, S. Abbadi, E. Rampazzo et al., "Intratumoral hypoxic gradient drives stem cells distribution and MGMT expression in glioblastoma," STEM CELLS, vol. 28, no. 5, pp. 851-862, 2010.

[119] Z. Li, S. Bao, Q. Wu et al., "Hypoxia-inducible factors regulate tumorigenic capacity of glioma stem cells," Cancer Cell, vol. 15, no. 6, pp. 501-513, 2009.

[120] S. Seidel, B. K. Garvalov, V. Wirta et al., "A hypoxic niche regulates glioblastoma stem cells through hypoxia inducible factor 2a," Brain, vol. 133, no. 4, pp. 983-995, 2010.

[121] J. Kolenda, S. S. Jensen, C. Aaberg-Jessen et al., "Effects of hypoxia on expression of a panel of stem cell and chemoresistance markers in glioblastoma-derived spheroids," Journal of Neuro-Oncology, vol. 103, no. 1, pp. 43-58, 2011.

[122] A. Eramo, L. Ricci-Vitiani, A. Zeuner et al., "Chemotherapy resistance of glioblastoma stem cells," Cell Death and Differentiation, vol. 13, no. 7, pp. 1238-1241, 2006.

[123] L. Shi, S. Zhang, K. Feng et al., "MicroRNA-125b-2 confers human glioblastoma stem cells resistance to temozolomide through the mitochondrial pathway of apoptosis," International Journal of Oncology, vol. 40, no. 1, pp. 119-129, 2012.

[124] P. Brescia, B. Ortensi, L. Fornasari, D. Levi, G. Broggi, and G. Pelicci, "CD133 is essential for glioblastoma stem cell maintenance," STEM CELLS, vol. 31, no. 5, pp. 857-869, 2013.

[125] I. Shibahara, Y. Sonoda, R. Saito et al., "The expression status of CD133 is associated with the pattern and timing of primary glioblastoma recurrence," Neuro-Oncology, vol. 15, no. 9, pp. 1151-1159, 2013.

[126] C.-H. Wang, S.-H. Chiou, C.-P. Chou, Y.-C. Chen, Y.-J. Huang, and C.-A. Peng, "Photothermolysis of glioblastoma stem-like cells targeted by carbon nanotubes conjugated with CD133 monoclonal antibody," Nanomedicine: Nanotechnology, Biology, and Medicine, vol. 7, no. 1, pp. 69-79, 2011.

[127] D. R. Emlet, P. Gupta, M. Holgado-Madruga et al., "Targeting a glioblastoma cancer stem-cell population defined by EGF receptor variant III," Cancer Research, vol. 74, no. 4, pp. 12381249, 2014.

[128] Y.-J. Boo, J.-M. Park, J. Kim et al., "L1 expression as a marker for poor prognosis, tumor progression, and short survival in patients with colorectal cancer," Annals of Surgical Oncology, vol. 14, no. 5, pp. 1703-1711, 2007.

[129] S. Izumoto, T. Ohnishi, N. Arita, S. Hiraga, T. Taki, and T. Hayakawa, "Gene expression of neural cell adhesion molecule L1 in malignant gliomas and biological significance of L1 in glioma invasion," Cancer Research, vol. 56, no. 6, pp. 1440-1444, 1996.

[130] S. Bao, Q. Wu, Z. Li et al., "Targeting cancer stem cells through L1CAM suppresses glioma growth," Cancer Research, vol. 68, no. 15, pp. 6043-6048, 2008.

[131] H. S. Günther, N. O. Schmidt, H. S. Phillips et al., "Glioblastoma-derived stem cell-enriched cultures form distinct subgroups according to molecular and phenotypic criteria," Oncogene, vol. 27, no. 20, pp. 2897-2909, 2008.
[132] A. T. Ogden, A. E. Waziri, R. A. Lochhead et al., "Identification of A2B5+CD133- tumor-initiating cells in adult human gliomas," Neurosurgery, vol. 62, no. 2, pp. 505-515, 2008.

[133] N. Gaiano and G. Fishell, "The role of notch in promoting glial and neural stem cell fates," Annual Review of Neuroscience, vol. 25, no. 1, pp. 471-490, 2002.

[134] X. Fan, W. Matsui, L. Khaki et al., "Notch pathway inhibition depletes stem-like cells and blocks engraftment in embryonal brain tumors," Cancer Research, vol. 66, no. 15, pp. 7445-7452, 2006.

[135] O. A. Guryanova, Q. Wu, L. Cheng et al., "Nonreceptor tyrosine kinase BMX maintains self-renewal and tumorigenic potential of glioblastoma stem cells by activating STAT3," Cancer Cell, vol. 19, no. 4, pp. 498-511, 2011.

[136] M. Kortylewski, M. Kujawski, A. Herrmann et al., “Toll-like receptor 9 activation of signal transducer and activator of transcription 3 constrains its agonist-based immunotherapy," Cancer Research, vol. 69, no. 6, pp. 2497-2505, 2009.

[137] A. Herrmann, G. Cherryholmes, A. Schroeder et al., "TLR9 is critical for glioma stem cell maintenance and targeting," Cancer Research, vol. 74, no. 18, pp. 5218-5228, 2014.

[138] A. S. Chung and N. Ferrara, "Developmental and pathological angiogenesis," Annual Review of Cell and Developmental Biology, vol. 27, pp. 563-584, 2011.

[139] C. Calabrese, H. Poppleton, M. Kocak et al., "A perivascular niche for brain tumor stem cells," Cancer Cell, vol. 11, no. 1, pp. 69-82, 2007.

[140] B. Badie and J. Schartner, "Role of microglia in glioma biology," Microscopy Research and Technique, vol. 54, no. 2, pp. 106-113, 2001.

[141] S. F. Hussain, D. Yang, D. Suki, E. Grimm, and A. B. Heimberger, "Innate immune functions of microglia isolated from human glioma patients," Journal of Translational Medicine, vol. 4, no. 1, article 15, 2006.

[142] B. C. Kennedy, L. M. Maier, R. D’Amico et al., "Dynamics of central and peripheral immunomodulation in a murine glioma model," BMC Immunology, vol. 10, no. 1, article 11, 2009.

[143] W. Li and M. B. Graeber, "The molecular profile of microglia under the influence of glioma," Neuro-Oncology, vol. 14, no. 8, pp. 958-978, 2012.

[144] S. Wagner, S. Czub, M. Greif et al., "Microglial/macrophage expression of interleukin 10 in human glioblastomas," International Journal of Cancer, vol. 82, no. 1, pp. 12-16, 1999.

[145] S. M. Pyonteck, L. Akkari, A. J. Schuhmacher et al., "CSF-1R inhibition alters macrophage polarization and blocks glioma progression," Nature Medicine, vol. 19, no. 10, pp. 1264-1272, 2013.

[146] W. Zhou, S. Q. Ke, Z. Huang et al., "Periostin secreted by glioblastoma stem cells recruits M2 tumour-associated macrophages and promotes malignant growth," Nature Cell Biology, vol. 17, no. 2, pp. 170-182, 2015. 

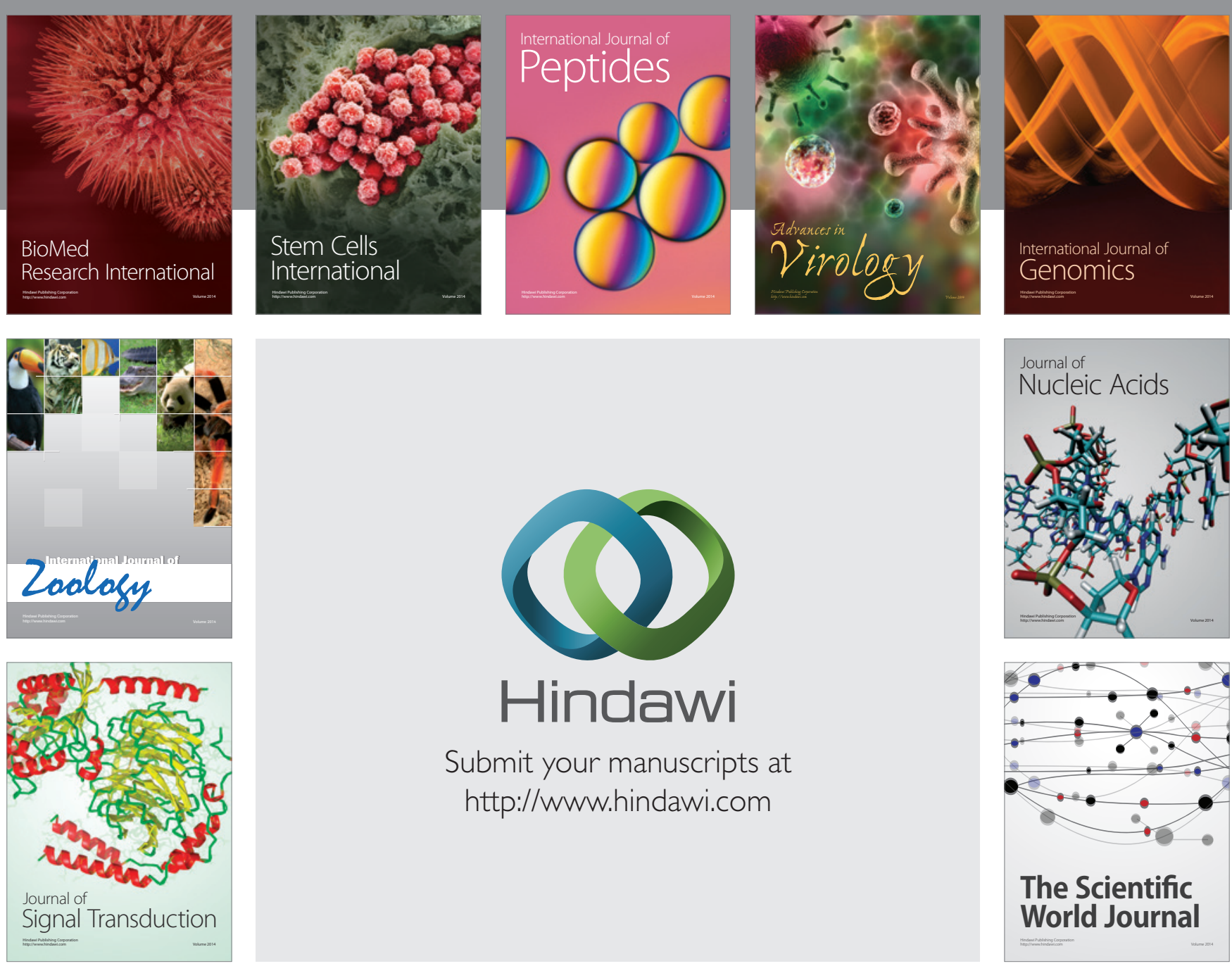

Submit your manuscripts at

http://www.hindawi.com
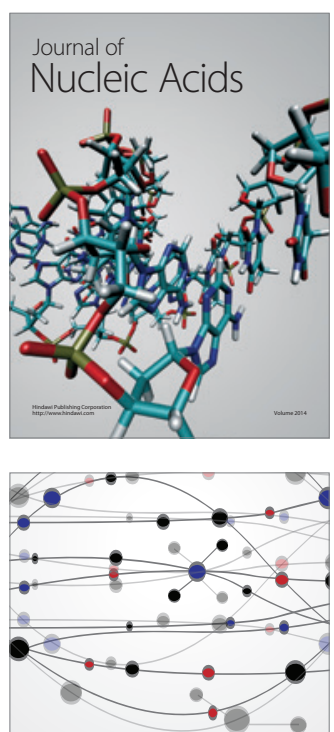

The Scientific World Journal
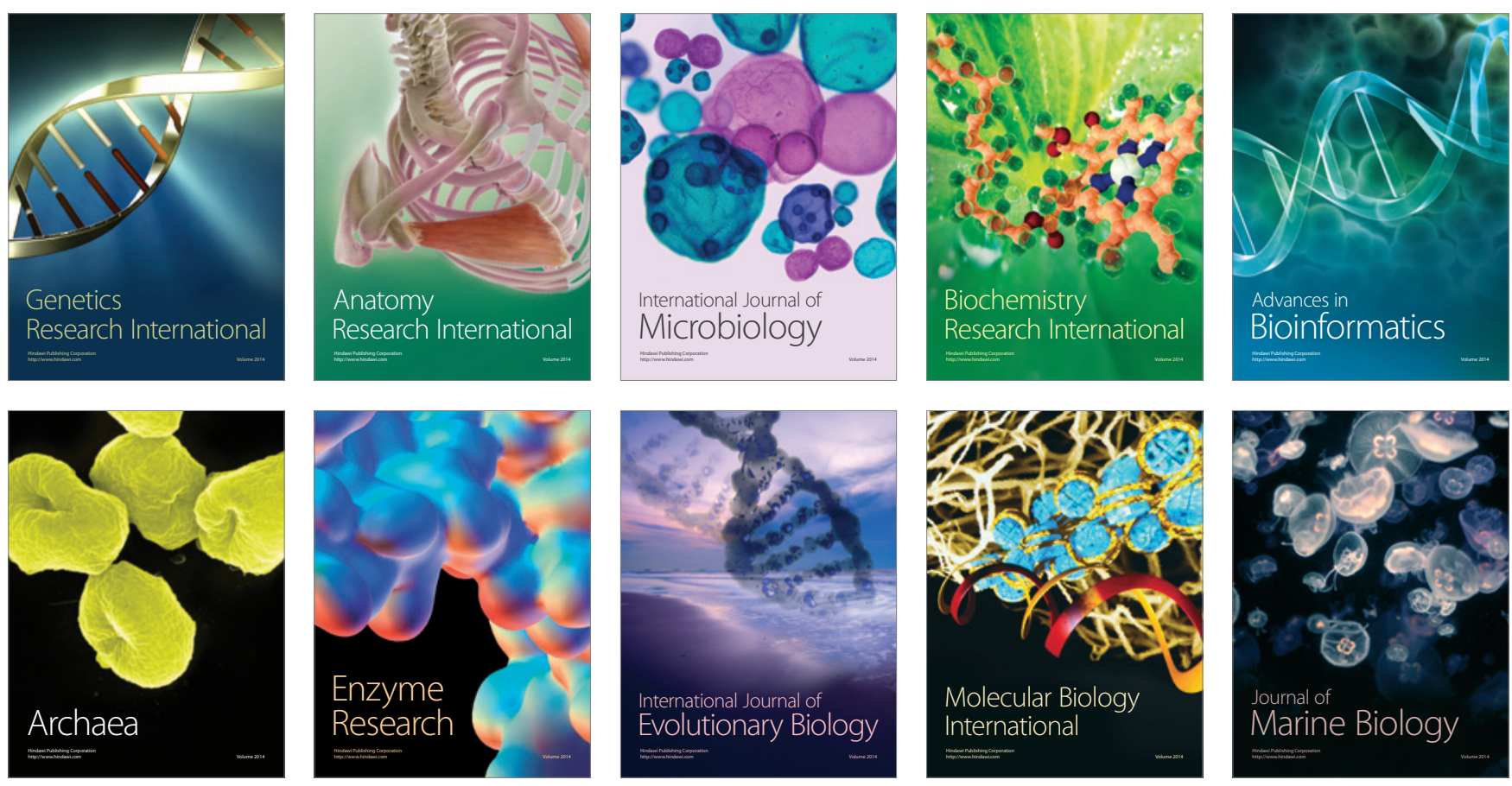